\title{
Evaluation of volumetric measurements on CBCT images using stafne bone cavities as an example
}

\author{
Mehmet-Zahit Adisen ${ }^{1}$, Selmi Yılmaz ${ }^{2}$, Melda Misirlioglu ${ }^{3}$, Fethi Atil ${ }^{4}$ \\ ${ }^{1}$ Lecturer (PhD). Department of Oral and Maxillofacial Radiology. Faculty of Dentistry, Kirıkkale University, Kirıkkale, Tur- \\ key \\ ${ }^{2}$ Research Assistant (PhD). Department of Oral and Maxillofacial Radiology. Faculty of Dentistry, Kirıkkale University, \\ Kirıkkale, Turkey \\ ${ }^{3}$ Assistant Professor (PhD). Department of Oral and Maxillofacial Radiology. Faculty of Dentistry, Kirıkkale University, \\ Kir1kkale, Turkey \\ ${ }^{4}$ Assistant Professor (PhD). Department of Oral and Maxillofacial Surgery. Faculty of Dentistry, Kirıkkale University, Kirıkkale, \\ Turkey
}

Correspondence:

Kırlkkale University

Faculty of Dentistry

Department of Oral and Maxillofacial Radiology

Kirlkkale, Turkey

mzahit@hotmail.com

Received: $24 / 01 / 2015$

Accepted: $25 / 04 / 201$

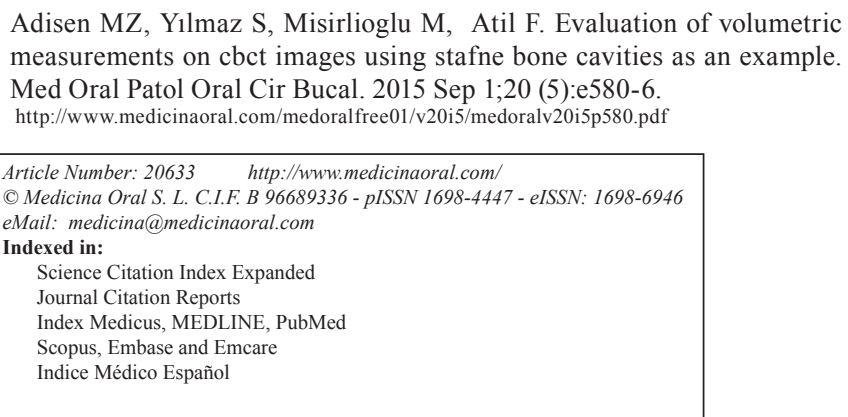

\begin{abstract}
Background: The aim of the present study is to evaluate the efficacy of CBCT in volume measuring using Stafne Bone Cavities (SBC) as an example.

Material and Methods: The study was conducted with 14 subjects with SBC detected on panoramic radiographs. In order to evaluate lesions volumetric dimensions, CBCT images for each patient were captured. Files in Digital Imaging and Communications in Medicine (DICOM) format were transferred into a medical image processing program (ITK-SNAP 2.4.0) and volume in $\mathrm{mm}^{3}$ of the cavities were measured using semi-automatic segmentation procedure by 2 observers blinded to each other over a one-month period. Inter-reliability of volumetric measurements between observers was compared. SBCs relation to mandibular canal was also examined and three types of relation were observed; type 1: mandibular canal is separated from the SBC, type 2: mandibular canal is in contact with SBC, type 3: mandibular canal goes through the SBC.

Results: There were 12 males and 2 females who had SBC in this study (age range: 37-73, mean age: 55.3 years). The total volume of SBC in patients ranged from $160 \mathrm{~mm}^{3}$ to $520 \mathrm{~mm}^{3}$ (mean: $361.7 \mathrm{~mm}^{3}$ ). There was no significant difference between observers for volume measurements $(p>0.05)$. According to relationship of SBC with mandibular canal, most SBCs were Type 1 (64.3\%) followed by type 3 (21.4 \%) and type 2 (14.3 \%). Pearson correlation coefficient shows a positive correlation between lesions volumetric size and relation with mandibular canal (pearson correlation $=0.54$, sig $<0.05$ ) .
\end{abstract}


Conclusions: Based on the results of this preliminary study, CBCT was considered to be an effective radiographic technic for measuring volumetric sizes of SBCs. However further studies with larger sample sizes are needed to prove the usefulness of $\mathrm{CBCT}$ in volume measurements.

Key words: Stafne bone cavity, CBCT, volumetric measurements, image segmentation.

\section{Introduction}

Volumetric measurements in the field of dentomaxillofacial radiology are usually made for detecting upper airway or sinonasal complex volume (1). They can also be used for evaluating volumetric size of various bone lesions including; periapical abscess, cysts and tumors (2). Detecting the volume of a lesion becomes important especially comparing the lesions dimensions with the follow-up radiographies. It is also important when reconstructive surgery with bone grafting is planned (3). When surgical access is necessary, quality imaging is critical to localize the lesion and its proximity to important structures. While the information gleaned from a dental radiograph is substantial, there are limitations associated with the use of a two-dimensional image. Therefore, an imaging modality with three- dimensional (3D) capability is essential to enhance diagnosis and treatment (4). The introduction of cone beam computed tomography (CBCT) has initiated a new era in the field of dentomaxillofacial radiology owing to the acquisition of large data volume in a short scan-time and at low radiation dose. CBCT scans produce reconstructed multiplanar images that allow the clinician to assess the area of interest three-dimensionally. It provides clear images of high-contrasted structures and is extremely useful for evaluating bone pathologies (5).

Many studies investigating dimensional accuracy of CBCT quote linear or angular measurements and they usually employed methods using calibration cubes, spherical phantoms of known size, calculating volume from manual linear readings or simulated lesions $(2,6,7)$. Currently, there are only a few studies confirming the accuracy of CBCT to measure lesions in bone volumetrically (5).

Stafne Bone Cavity (SBC) is a homogeneous radiolucent lesion with well-defined borders usually located in the mandible below the inferior dental canal, a suitable candidate for volumetric measurements on CBCT images. It was stated that $\mathrm{CBCT}$ provides accurate information on the shape, location and size of SBC (8). In the present study, we aimed to evaluate the efficacy of CBCT for volumetric measurement using SBCs as an example.

\section{Material and Methods}

The study was conducted with 14 subjects selected from among 16782 patients who underwent clinical and pan- oramic radiographic examinations in the Department of Oral and Maxillofacial Radiology between January 2012 and October 2013. Panoramic radiographs were obtained using OP 200D (Instrumentarium Dental, Tuusula, Finland) by two radiography technicians both of whom had a minimum working experience of 5 years. (57-85 kVp, 2-16 mA, and $8 \mathrm{sec}$.)

Panoramic radiographs were examined by three oral radiologists and one oral surgeon who were able to use brightness and contrast tools to optimize their diagnosis and classify SBC on 14 cases using the same criteria under optimum viewing conditions. All of the patients agreed to participate in the study and signed an informed consent form. This study had ethical approval by the Research Committee of the Faculty of Dentistry at Kirıkkale University and is in compliance with the Helsinki Declaration.

An ID number has been assigned for every patient; who has been associated with collected data pertaining to demographic information (age, sex), medical and dental history, and the location of the cavity.

In order to evaluate $\mathrm{SBCs}$ volumetric dimensions, $\mathrm{CBCT}$ images for each patient were captured using PaxUni 3D (Vatech, Seoul, Korea) at the following settings: 50-90 $\mathrm{kVp}, 4-10 \mathrm{~mA}, 10 \mathrm{sec}$ exposure time, and a $50 \times 50 \mathrm{~mm}$ field of view (FOV) size. Obtained image files in Digital Imaging and Communications in Medicine (DICOM) format were transferred into a medical image processing program (ITK-SNAP 2.4.0) and volume in $\mathrm{mm}^{3}$ of the cavities were measured using semi-automatic segmentation procedure (0.2-mm slice thickness).

When measuring volumes in ITK-SNAP software, the regions of interest were determined in square areas selecting the most exterior point of the concavities in all three planar views (coronal, axial and sagittal). The "Segment 3D" tool was used and an appropriate threshold level was adjusted. Then, the reference points were selected on the cavity for semiautomatic segmentation. When the "Start segmentation" tool was used, the software automatically segmented the cavity starting from the reference points using the contrast differences on the greyscale images. Thus the $3 \mathrm{D}$ model and the volume in $\mathrm{mm}^{3}$ were obtained with the software (Fig. 1). All of the measurements were made by 2 observers (experienced in image segmentation) blinded to each other over a one-month period. 2 maxillofacial radiologists 


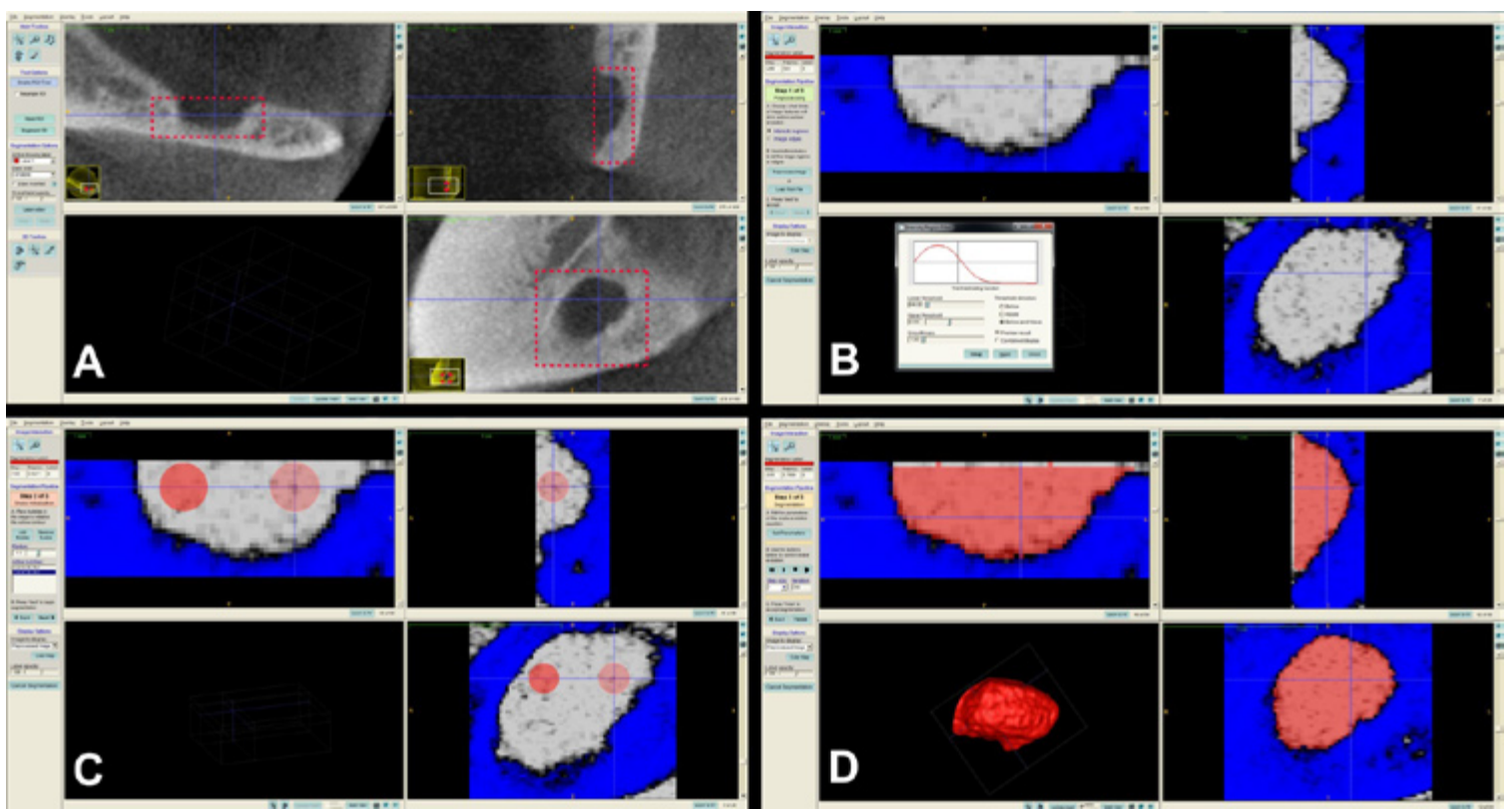

Fig. 1. In ITK-SNAP software, region of interest was determined in a square area in all three planes of views (a). "Segment 3D" tool was used and same threshold level with the original software (Ez3D2009) was determined (b). Then the reference points were selected on the cavity for semiautomatic segmentation (c). When the "Start segmentation" tool was used, the software automatically segmented the cavity starting from the reference points using the contrast differences on the greyscale images. Thus the 3D model and the volume in $\mathrm{mm} 3$ were obtained with the software (d).

separately evaluated 14 CBCT images on a computer monitor (Philips 273EQH 27-inch LED monitor with $1920 \times 1080$ resolution), under ambient lighting conditions. The examiners were left to choose their own threshold settings based on the best visualization of the cavities. Intra-observer reproducibility was assessed by having the observers re-evaluate 5 randomly selected CBCT images after an interval of one week.

Following the volume measurements, SBCs relation to mandibular canal was also examined and three types of relation were observed (9) (Fig. 2):

Type 1: Mandibular canal is separated from the SBC.

Type 2: Mandibular canal is in contact with SBC.

Type 3: Mandibular canal goes through the SBC.

All the obtained data were entered in the Statistical Package for Social Sciences Program (SPSS 11.5). A Nonparametric Kruskal-Wallis test was used to compare inter-reliability of volumetric measurements between observers $(p<0.05)$. Cohen's Kappa and Cronbach's Alpha tests were used to test intra-observer agreement. Also correlation between lesions volume and proximity with mandibular canal was evaluated using Pearson's correlation coefficient.

\section{Results}

There were 12 males and 2 females who had SBC in this study (age range: $37-73$, mean age: 55.3 years).

Cohen's Kappa and Cronbach's alpha values showed excellent intra-observer agreement (kappa > 90 and alpha
$>93$ ) for both observers. Inter-reliability of volumetric measurements between observers was shown in table 1 . There was no significant difference between observers for volume measurements $(p>0.05)$. The total volume of SBC ranged from $160 \mathrm{~mm}^{3}$ to $520 \mathrm{~mm}^{3}$ (mean: 361.7 $\mathrm{mm}^{3}$ ) (Table 2). All the SBCs were posterior lingual variant. Bilateral presentation was not noted. Whereas 14 patients had unilateral SBC; $8(54.1 \%)$ were left and $6(45.9 \%)$ were on the right side. According to relationship of SBC with mandibular canal, most SBCs were Type $1(64.3 \%)$ followed by type $3(21.4 \%)$ and type $2(14.3 \%)$. Pearson correlation coefficient shows a positive correlation between lesions volumetric size and relation with mandibular canal (pearson correlation $=0.54$, sig $<0.05)$.

\section{Discussion}

The aim of the present study was to evaluate the efficacy of CBCT in volume measuring using Stafne Bone Cavities' (SBC) as an example. No significant differences were found between observers for volume measurements and the total volume of SBC found to be ranged from $160 \mathrm{~mm}^{3}$ to $520 \mathrm{~mm}^{3}$ (mean: $361.7 \mathrm{~mm}^{3}$ ). In the previous studies diameter and depth of the cavities were measured and it was reported that SBCs sizes varied 1 to $3 \mathrm{~cm}$ in diameter (10). The depth and width of the concavities averaged 7,9 and 16,3 $\mathrm{mm}$ respectively (11). To the best of our knowledge, this is the first study measuring volumetric sizes of SBCs. 

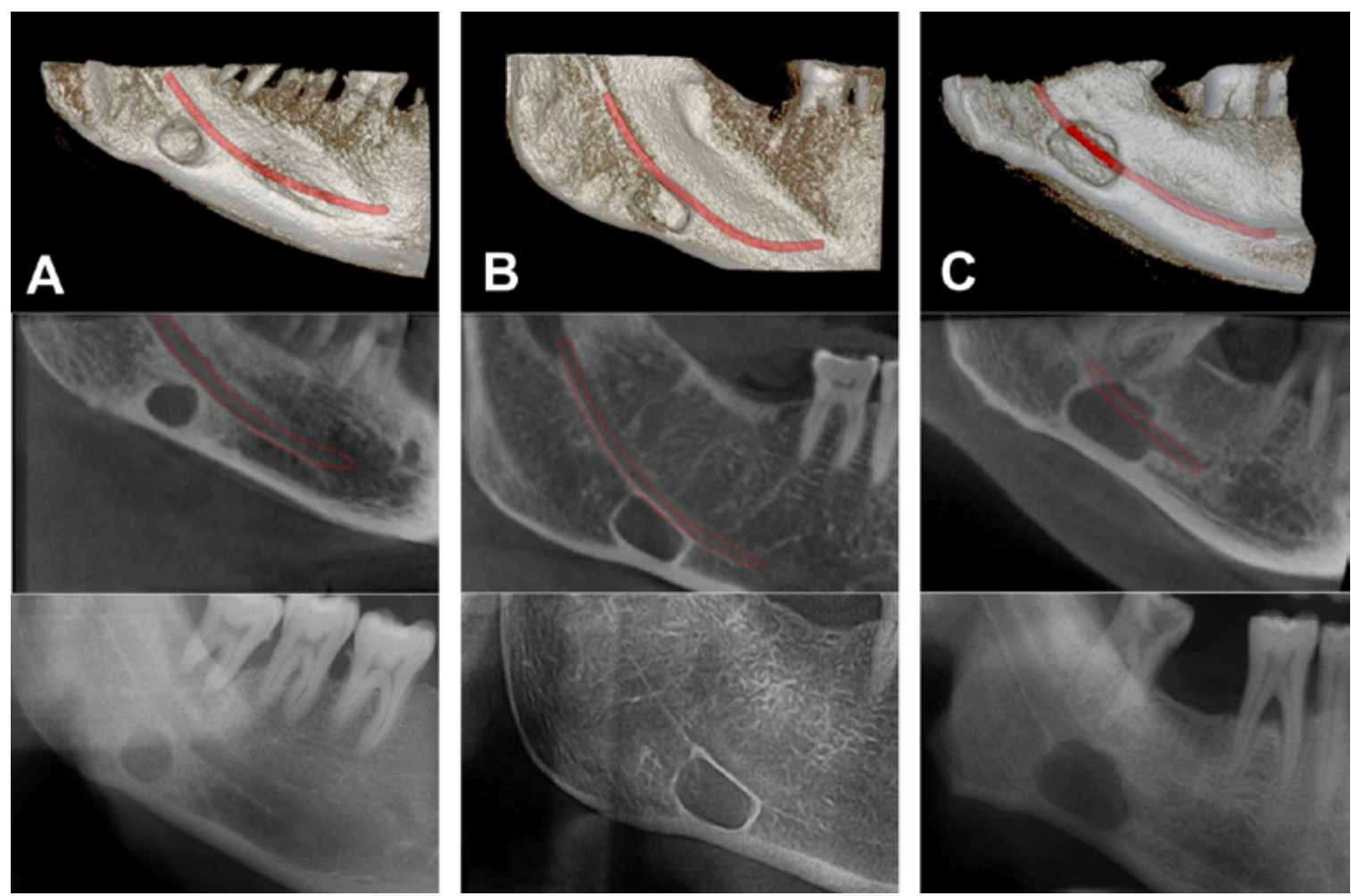

Fig. 2. This figures show the SBCs relation to mandibular canal on CBCT images (3D and sagittal view) and panoramic radiographs. Three types of relation were observed.

a) Mandibular canal is separated from the SBC.

b) Mandibular canal is in contact with SBC.

c) Mandibular canal goes through the SBC.

Table 1. Inter-reliability of volumetric measurements between observers.

\begin{tabular}{|l|c|c|c|c|c|}
\hline & N & Mean & S.D. & Chi-S & $\boldsymbol{P}$ \\
\hline Observer 1 & 14 & 360.8 & 36.95 & & \\
Observer 2 & 14 & 362.7 & 29.36 & 0.011 & 0.92 (ns) \\
Total & 28 & 360.1 & 22.58 & & \\
\hline
\end{tabular}

ns: Not significant.

Volumetric measurements can be performed using various PC programs such as Mimics, Dolphin, ITK-Snap etc. Weissheimer at al. compared the accuracy of 6 different imaging softwares (Mimics, ITK-Snap, OsiriX, Dolphin3D, InVivo Dental, Ondemand3D) for 3-dimensional analysis of the upper airway and found that all 6 imaging software programs were reliable but had errors in the volume segmentations of the oropharynx. Mimics, Dolphin3D, ITK-Snap, and OsiriX were similar and more accurate than InVivo Dental and Ondemand3D for upper airway assessment (12). Additionally Alhowalia et $a l$. tested the accuracy of CBCT for volumetric measurement of simulated periapical lesions and concluded that $\mathrm{CBCT}$ is an accurate means of making volumetric meas-
Table 2. SBC characteristics of 14 cases.

\begin{tabular}{|c|c|c|c|c|c|}
\hline $\begin{array}{c}\text { Patient } \\
\text { no }\end{array}$ & Age & Sex & Laterality & $\begin{array}{c}\text { Relation to } \\
\text { Mandibular } \\
\text { Canal }\end{array}$ & $\begin{array}{c}\text { Volume } \\
\text { (Mean) }\end{array}$ \\
\hline 1 & 62 & $\mathrm{M}$ & Left & 1 & 286.25 \\
\hline 2 & 38 & $\mathrm{M}$ & Left & 1 & 350 \\
\hline 3 & 73 & $\mathrm{M}$ & Right & 2 & 446.25 \\
\hline 4 & 59 & $\mathrm{M}$ & Left & 1 & 160.75 \\
\hline 5 & 37 & $\mathrm{M}$ & Left & 1 & 501.25 \\
\hline 6 & 50 & $\mathrm{M}$ & Right & 2 & 447.5 \\
\hline 7 & 55 & $\mathrm{M}$ & Left & 1 & 327.5 \\
\hline 8 & 43 & $\mathrm{M}$ & Left & 3 & 498.75 \\
\hline 9 & 73 & $\mathrm{M}$ & Right & 1 & 270 \\
\hline 10 & 53 & $\mathrm{~F}$ & Left & 1 & 226.25 \\
\hline 11 & 68 & $\mathrm{M}$ & Right & 3 & 402.5 \\
\hline 12 & 64 & $\mathrm{~F}$ & Right & 3 & 520 \\
\hline 13 & 54 & $\mathrm{M}$ & Right & 1 & 360,5 \\
\hline 14 & 45 & $\mathrm{M}$ & Left & 2 & 266.25 \\
\hline
\end{tabular}

1: Mandibular canal is separated from the SBC.

2: Mandibular canal is in contact with SBC.

3: Mandibular canal goes through the SBC.

urements of artificially created cavities within bone with the use of appropriate software and training (2).

Currently, there are few studies confirming the accuracy of CBCT to measure lesions in bone volumetrically (5). Previous studies have employed methods using 
calibration cubes, spherical phantoms of known size or calculating volume from manual linear readings $(4,13)$. However it is uncertain whether this same method would work for lesions of irregular shape. Alhowalia et al. used manual tracing of simulated bone lesions on bovine bones and found that measurements were highly accurate and inter-observer variability to be relatively low. They emphasized that manual tracing is more time consuming than software guided segmenting and suggested that it would be beneficial to study further the accuracy of software-guided segmenting (2).

When using automated segmenting method it is important to select appropriate threshold level and borders of the lesion being segmenting. Otherwise the volume of the lesions may be measured incorrectly. Hence the observers should get training by a reference standard examiner (14). In the present study observers were experienced in image segmentation and got training before.

The typical radiographic presentation of a SBC is of an elliptical, homogeneous radiolucency with a welldefined border sited below the inferior alveolar canal, often involving the lower border of the mandible. Extension of the cavity above the inferior dental nerve was rarely reported (9). Oikarinen and Julku found three cases immediately adjacent to the mandibular canal in their study of 10000 panoramic radiographs (15). In the present study we found that mandibular canal was going through the cavity in 3 cases (Fig. 3) and in contact with the cavity in 2 cases. Also larger lesions were found to be involved with mandibular canal more likely than smaller lesions. Uemura refers to a case in Japanese literature where on the radiograph, the neurovascular bundle ran centrally, but at surgery a completely closed cavity without a lingual defect was found (16). A key to anatomic feature of SBC is that the mandibular concavity always is open on its lingual face (17). Therefore this case cannot be diagnosed as a SBC due to lack of lingual defect. The advanced imaging techniques become important in such cases. The panoramic and the oblique lateral radiographs are insufficient to verify the integrity of the bone margins (9). Hence CT, MRI and sialography techniques have been used to achieve a final diagnosis of SBC (18). Sisman et al. reported that CT scans are usually sufficient for definitive diagnosis of SBCs (19). However Segev et al. mentioned that CT should be supported with MRI to identify the content of the cavity (20). In addition, Smith et al. reported that increased ionized radiation exposure and possible contrast allergy are disadvantages of CT and they concluded that MRI is the most useful diagnostic tool for detecting the content and extent of SBC (21). However, its high cost and relatively long imaging time limit the routine use of MRI. Sialography is also a diagnostic technique that can be used to determine whether glandular tissue exist in the cavity (22). On the other hand this procedure is invasive and uncomfortable for patients. Additionally allergy - like hypersensitivity reactions have been observed after use of X-ray contrast media. Serious events such as angioedema, subglottic oedema, bronchospasm and allergic shock are possible (23). Also sialographic evaluation of anterior variants of SBC is relatively hard to perform owing to multiple ducts in the sublingual gland (19). Recently, CBCT with high-level spatial resolution has been used for diagnostic imaging of the oral and maxillofacial regions and various anatomical structures. Owing to its lower radiation exposure, low cost and higher speed relative to conventional CT and MRI has made CBCT a valuable tool for diagnosis of various

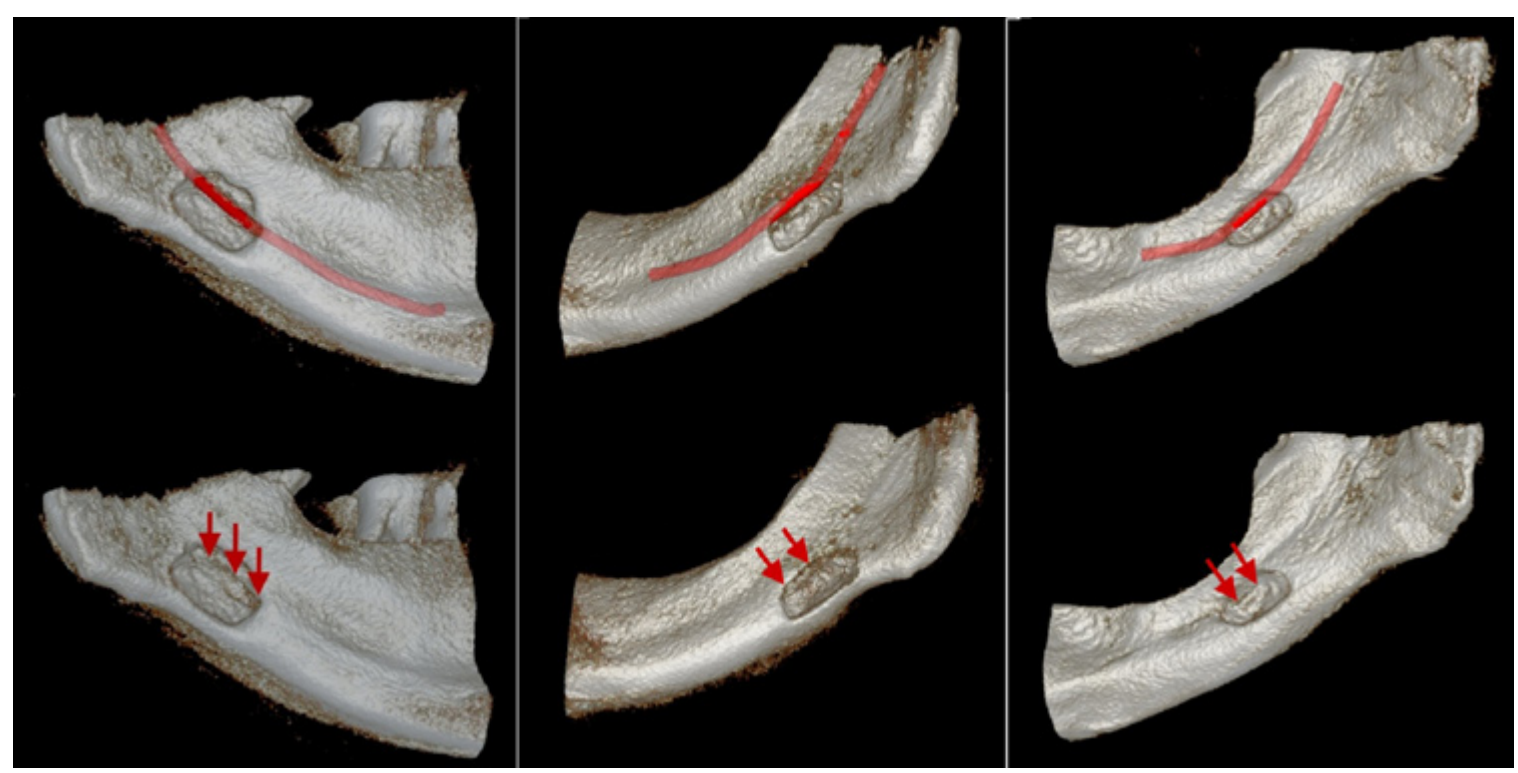

Fig. 3. 3D images of 3 cases that mandibular canal is going through the cavity. 
bone lesions (24). Additionally considering the ALARA (As Low As reasonably Achievable) different FOV sizes can be selected for different radiographic modalities in many CBCT devices. Matching the FOV to the area of interest can optimize the effective dose $(22 \mathrm{~cm}$ FOV $-206.2 \mu \mathrm{Sv}, 13 \mathrm{~cm}$ FOV- $133.9 \mu \mathrm{Sv}$ and $6 \mathrm{~cm}$ FOV- 96.2 $\mu$ Sv. 2007 International Commission on Radiological Protection tissue-weighting factors for the i-CAT Classic CBCT machine) (25). Katz et al. suggested that CBCT provided detailed information about the definitive diagnosis of SBC (26). Sisman et al. suggested that since CBCT has low radiation dose and shows fine details and superior features in distinguishing suspicious radiolucent lesions of the mandible, it might be used for diagnosis of SBC (19).

The radiographic diagnosis of SBCs has included benign salivary gland tumours, neurogenic tumours, hemangioma, myxoma, central giant cell lesion, odontogenic cyst, simple bone cyst, ameloblastoma, fibroosseous lesions, multiple myeloma, eosinophilic granuloma and metastatic disease (27). The literature featured a case of ossifying fibroma presenting as SBC (28). Some studies showed that SBCs were not developmental lesions and continued to grow gradually and become larger over a long period of observation. In addition, superimposed pathology, such as pleomorphic adenoma, can develop in the entrapped salivary gland (29). In the present study we did not aim to observe if any alterations become in SBCs over time, however with the guidance of the volumetric measurements; changes in the volumetric dimensions can be observed through further studies. Management of SBC should be conservative; a long term radiographic follow-up is required. Surgical exploration and biopsy should only be performed when the diagnosis is uncertain or an unusually severe pathology is suspected.

In conclusion, based on the results of this preliminary study, CBCT was considered to be an effective radiographic technic for measuring volumetric sizes of SBCs. However further studies with larger sample sizes are needed to prove the usefulness of CBCT in volume measurements when reconstructive surgery or followup study will be performed on various bone lesions.

\section{References}

1. Alsufyani NA, Flores-Mir C, Major PW. Three-dimensional segmentation of the upper airway using cone beam CT: a systematic review. Dentomaxillofac Radiol. 2012;41:276-84.

2. Ahlowalia MS, Patel S, Anwar HM, Cama G, Austin RS, Wilson $\mathrm{R}$, et al. Accuracy of CBCT for volumetric measurement of simulated periapical lesions. Int Endod J. 2013;46:538-46.

3. Economopoulos TL, Asvestas PA, Matsopoulos GK, Molnár B, Windisch P. Volumetric difference evaluation of registered threedimensional pre-operative and post-operative CT dental data. Dentomaxillofac Radiol. 2012;41:328-39.

4. Pinsky HM, Dyda S, Pinsky RW, Misch KA, Sarment DP. Accuracy of three-dimensional measurements using cone-beam CT. Dentomaxillofacial Radiol. 2006;35:410-6.
5. Agbaje JO, Jacobs R, Maes F, Michiels K, van Steenberghe D. Volumetric analysis of extraction sockets using cone beam computed tomography: a pilot study on ex vivo jaw bone. J Clin Periodontol. 2007;34:985-90.

6. Brown AA, Scarfe WC, Scheetz JP, Silveira AM, Farman AG. Linear accuracy of cone beam CT derived 3D images. Angle Orthodontist. 2009;79:150-7.

7. Damstra J, Fourie Z, Huddleston Slater JJ, Ren Y. Accuracy of linear measurements from cone-beam computed tomography-derived surface models of different voxel sizes. American Journal of Orthodontics and Dentofacial Orthopaedics. 2010;137:11-7.

8. Kopp S, Ihde S, Bienengraber V. Differential diagnosis of stafne idiopathic bone cyst with digital volume tomography (DVT). J Maxillofac Oral Surg. 2010;9:80-1.

9. Reuter I. An unusual case of Stafne bone cavity with extraosseous course of the mandibular neurovascular bundle. Dentomaxillofac Radiol. 1998;27:189-91.

10. Flores Campos PS, Oliveira JA, Dantas JA, de Melo DP, Pena N, Santos LA, et al. Stafne's defect with buccal cortical expansion: a case report. Int J Dent. 2010;2010:515931.

11. Ariji E, Fujiwara N, Tabata O, Nakayama E, Kanda S, Shiratsuchi $\mathrm{Y}$, et al. Stafne's bone cavity. Classification based on outline and content determined by computed tomography. Oral Surg Oral Med Oral Pathol. 1993;76:375-80.

12. Weissheimer A, Menezes LM, Sameshima GT, Enciso R, Pham $\mathrm{J}$, Grauer D. Imaging software accuracy for 3-dimensional analysis of the upper airway. Am J Orthod Dentofacial Orthop. 2012;142:80113.

13. Eggers G, Klein J, Welzel T, Mühling J. Geometric accuracy of digital volume tomography and conventional computed tomography. Br J Oral Maxillofac Surg. 2008;46:639-44.

14. Wang Y, He S, Guo Y, Wang S, Chen S. Accuracy of volumetric measurement of simulated root resorption lacunas based on cone beam computed tomography. Orthod Craniofac Res. 2013;16:169-76. 15. Oikarinen VJ, Julku M. An orthopantomographic study of developmental mandibular bone defects (Stafne's idiopathic bone cavities). Int J Oral Surg. 1974;3:71-6.

16. Uemura S, Fujishita M, Fuchihata H. Radiographic interpretation of so-called developmental defect of mandible. Oral Surg Oral Med Oral Pathol. 1976;41:120-8.

17. Bornstein MM, Wiest R, Balsiger R, Reichart PA. Anterior Stafne's bone cavity mimicking a periapical lesion of endodontic origin: report of two cases. Journal of Endodontics. 2009;35:1598-602.

18. Etöz M, Etöz OA, Şahman H, Şekerci AE, Polat HB. An unusual case of multilocular Stafne bone cavity. Dentomaxillofac Radiol. 2012;41:75-8.

19. Sisman Y, Miloglu O, Sekerci AE, Yilmaz AB, Demirtas O, Tokmak TT. Radiographic evaluation on prevalence of Stafne bone defect: a study from two centres in Turkey. Dentomaxillofac Radiol. 2012;41:152-8.

20. Segev Y, Puterman M, Bodner L. Stafne bone cavity--magnetic resonance imaging. Med Oral Patol Oral Cir Bucal. 2006;11:E345-7. 21. Smith MH, Brooks SL, Eldevik OP, Helman JI. Anterior mandibular lingual salivary gland defect: A report of a case diagnosed with cone-beam computed tomography and magnetic resonance imaging. Oral Surg Oral Med Oral Pathol Oral Radiol Endod. 2007;103:71-8.

22. Li B, Long X, Cheng Y, Wang S. Cone beam CT sialography of Stafne bone cavity. Dentomaxillofac Radiol. 2011;40:519-23.

23. Cockrell DJ, Rout PG. An adverse reaction following sialography. Dentomaxillofac Radiol. 1993;22:41-2.

24. Belmonte-Caro R, Velez-Gutierrez MJ, Garcia De La Vega-Sosa FJ, Garcia-Perla-Garcia A, Infante-Cossio PA, Diaz-Fernandez JM, et al. A Stafne's cavity with unusual location in the mandibular anterior area. Med Oral Patol Oral Cir Bucal. 2005;10:173-9.

25 . Hatcher DC. Operational principles for cone-beam computed tomography. JADA. 2010;141:3S-6S.

26. Katz J, Chaushu G, Rotstein I. Stafne's bone cavity in the anterior mandible: a possible diagnostic challenge. J Endod. 2001;27:304-7. 
27. Schneider T, Filo K, Locher MC, Gander T, Metzler P, Grätz KW, et al. Stafne bone cavities: systematic algorithm for diagnosis derived from retrospective data over a 5-year period. British Journal of Oral and Maxillofacial Surgery. 2014;52:369-74.

28. Parvizi F, Rout PG. An ossifying fibroma presenting as Stafne's idiopathic bone cavity. Dentomaxillofac Radiol. 1997;26:361-3.

29. Simpson W. A stafne's mandibular defect containing a pleomorphic adenoma: Report of case. J Oral Surg. 1965;23:553-6.

\section{Acknowledgments}

There are no acknowledgments. 